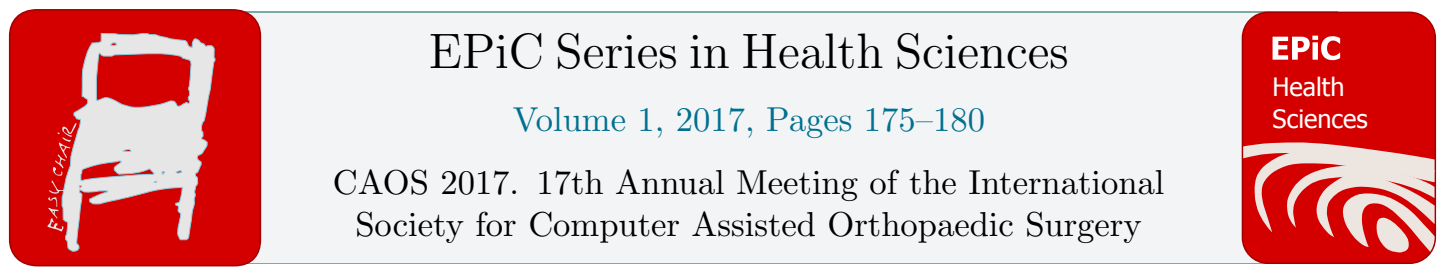

\title{
Multi-dimensional Range-of-Motion-based safe zone for patient-specific total hip arthroplasty
}

\author{
Juliana Hsu, Matías de la Fuente and Klaus Radermacher \\ Chair of Medical Engineering, Helmholtz Institute for Biomedical Engineering, RWTH Aachen \\ University, Germany \\ jhsu@hia.rwth-aachen.de
}

\begin{abstract}
Proper component alignment is crucial for a successful total hip arthroplasty (THA). Some studies found safe cup orientations and corresponding stem antetorsions based on a defined desired range of motion (ROM) suitable for activities of daily living. These studies either used complex and time consuming 3D simulations or more simple mathematical formulas which cannot be extended to combined motions.

With the method introduced in this work, any arbitrary motion can be applied. The ROM specified as the ROM of the femur relative to the pelvis is transformed into the ROM of the prosthesis neck relative to the cup for each cup orientation. For this transformation, the orientation and design of the stem are considered. The comparison of the neck and cup orientations is done using a 2D mapping of a 3D spherical surface which reduces the complexity of the calculation.

We found that the femoral antetorsion as well as the neutral stem flexion and adduction have an influence on the resulting safe zone. The result is not just a combined anteversion but a combined orientation. For validating the plausibility of the algorithm, the resulting safe zones are compared to literature. Same results can be achieved using the same input data. Using this technique, a patient-specific safe zone based on the ROM can be derived and adjusted to the stem orientation.
\end{abstract}

\section{Introduction}

Proper component alignment is crucial for a successful total hip arthroplasty (THA). Even when the cup is orientated according to the Lewinnek safe zone (Lewinnek, et al., 1978), many hips still dislocate (e.g. (Biedermann, et al., 2005; Abdel, et al., 2016)). This might be due to the fact, that the Lewinnek safe zone describes a range of cup orientations suitable for the average patient but does not consider any patient-specific nor functional parameters such as the range of motion (ROM), the pelvic tilt, or the orientation of the femoral component. 
Some studies used the ROM as the criterion for finding safe cup orientations. In the study by Widmer (Widmer \& Zurfluh, 2004), a desired ROM was defined and a 3D simulation model with a predefined implant design was used to simulate hip joint motions for a range of cup orientations. The parameters are summarized in Table 1. Impingements were detected and safe cup orientations were identified. A formula for calculating the optimal cup orientation which depends on the stem antetorsion was derived. A similar approach was introduced by Yoshimine (Yoshimine \& Ginbayashi, 2002; Yoshimine, 2006). A different desired ROM was defined (see Table 1) and impingement was calculated using a simple mathematical formula instead of using 3D computer simulation.

The drawback of the approach by Widmer is that the 3D simulation might be time consuming and for changed implant parameters, the simulation has to be repeated. Even though a combined motion consisting of for example flexion, adduction and internal rotation would be possible to simulate, it was not done in the study. The computation by the approach by Yoshimine is fast, however, with the introduced mathematical formula, extension to considering combined motion is not straight forward.

With the method introduced in this work, any arbitrary motion can be applied. Furthermore, parameters for stem orientation including not only antetorsion but also neutral stem flexion and adduction are considered.

\section{Materials and Methods}

Any motion of the femur can be described as a sequence of transformations $\mathrm{R}(\varphi, \alpha, \rho)$ consisting of a combination of flexion/extension $\varphi$, adduction/abduction $\alpha$ and internal/external rotation $\rho$. The convention recommended by the International Society of Biomechanics (ISB) (Wu, et al., 2002) was used. In order to determine whether a certain motion causes impingement or not, the corresponding neck orientations are calculated and compared to the limits of the cup. The entire workflow is shown in Figure 1. First, the femur ROM is converted to the neck ROM by applying a transformation describing the orientation of the femoral neck relative to the femur. This transformation depends on the antetorsion, the neck angle and neutral stem adduction as well as the neutral stem flexion. The limits of the cup can be described by the oscillation angle $\theta$ defined in (Yoshimine, 2006) which depends on the head-neck ratio. The comparison of the neck ROM with the cup limits can be done in $3 \mathrm{D}$ space or in $2 \mathrm{D}$ by mapping the $3 \mathrm{D}$ spherical surface into a $2 \mathrm{D}$ plane. The latter is less complex. The mapping can be done by simply reducing a transformation represented in $3 \mathrm{D} R(\varphi, \alpha, \rho)$ into a $2 \mathrm{D}$ point $\mathrm{p}(\varphi, \alpha)$. The cup limits are calculated for each head-neck ratio and mapped in 2D. For all cup orientations, the neck ROM is converted into the cup coordinate system and it is checked if all points of the neck ROM are inside the cup limits. This can be done using a pre-calculated map specifying whether a point is inside or outside of the cup limits. If all points are inside, the cup orientation is marked as safe (green) else it is marked as unsafe (red). Based on a variation of all parameters, a multi-dimensional safe zone is calculated.

For validating the plausibility of the algorithm, the resulting safe zones are compared to literature (test case 1). The same input data as in (Widmer \& Zurfluh, 2004) and (Yoshimine, 2006) (see Table 1) was used and the results are compared. Moreover, further desired ROMs from literature were used as input and the results are compared to each other (test case 2). The corresponding ROM limits (flexion/extension+adduction/abduction) and motion sequences of dislocation-prone motion were derived from the graphs published in the studies of (Turley, et al., 2011) and (Nadzadi, et al., 2003) manually. 


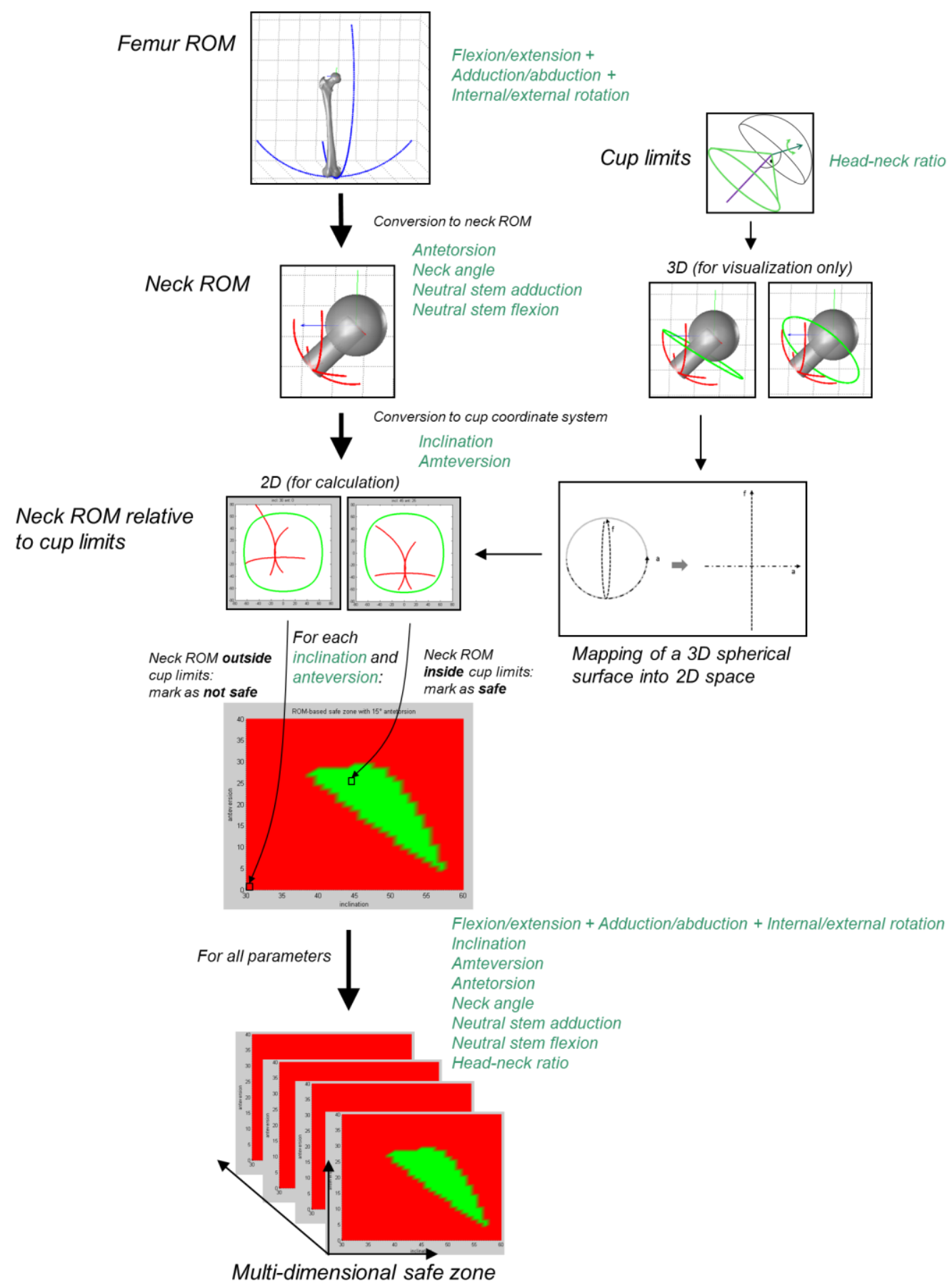

Figure 1: Calculation method for determine a range of motion based safe zone. 


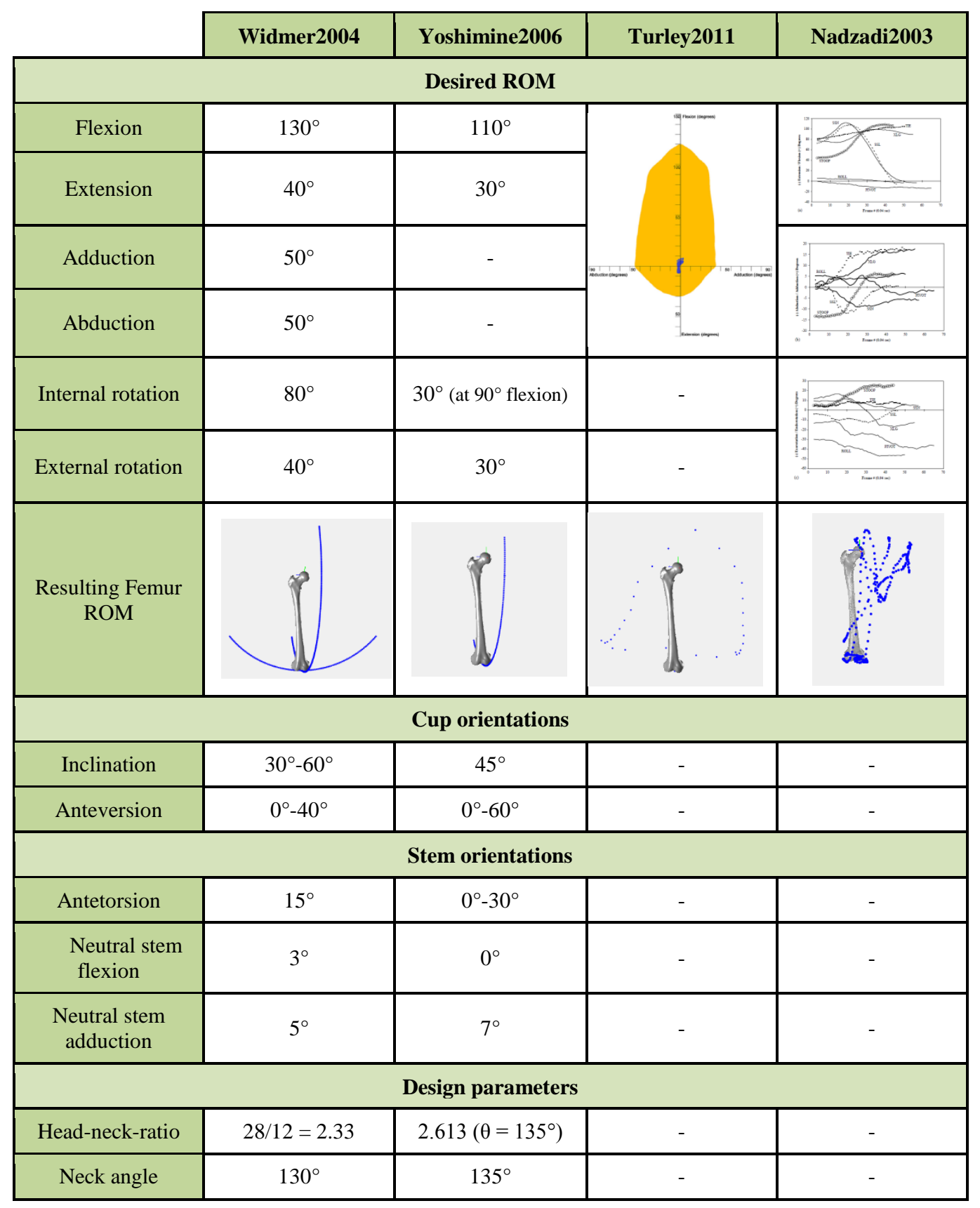

Table 1: Overview of range of motion-based safe zone calculation input. 


\section{Results}

The results are shown in Table 2. In test case 1, the resulting safe zones are the same as published in the corresponding studies. In test case 2 , it can be seen that the desired input ROM has a great influence on the resulting safe zone.

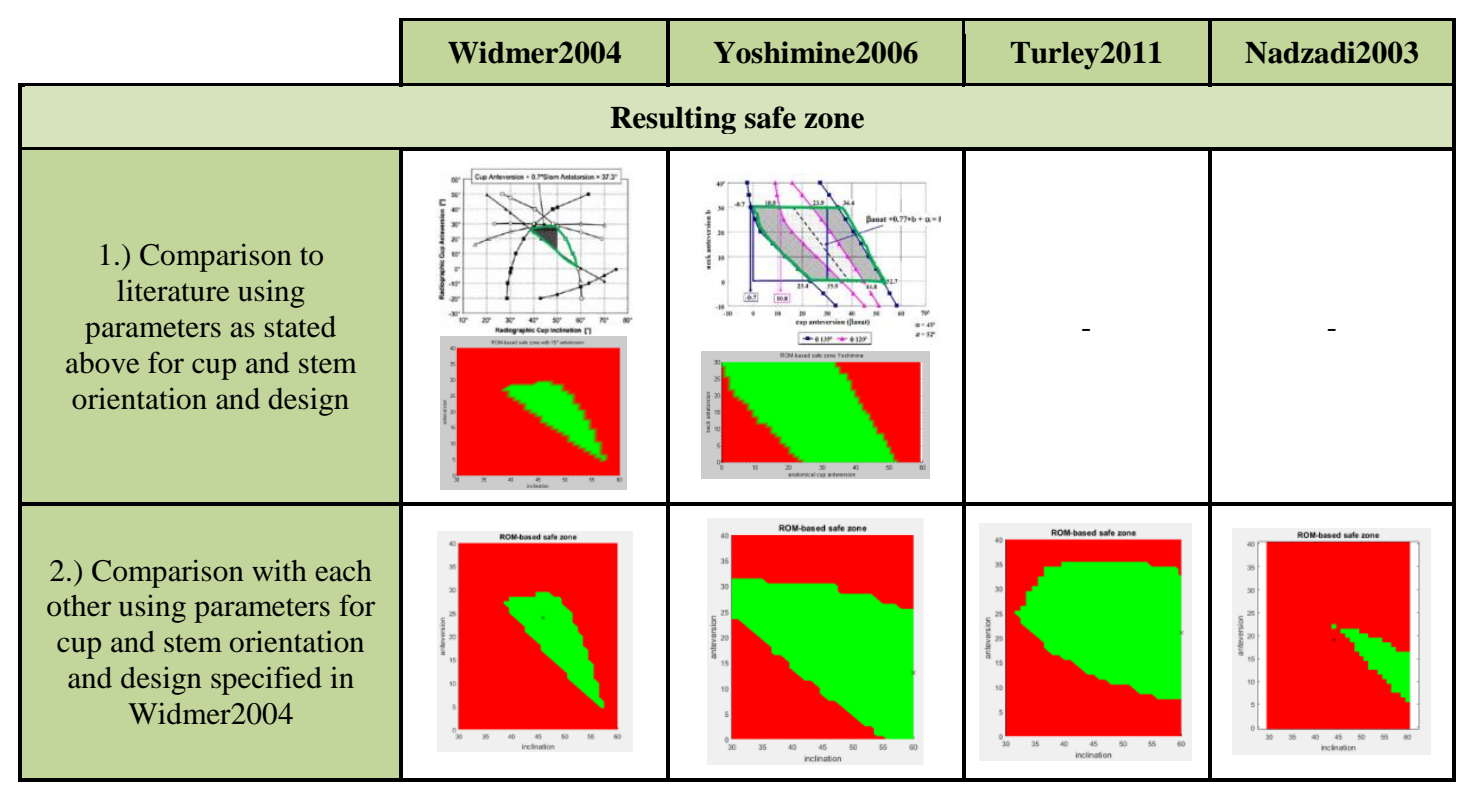

Table 2: Overview of range of motion-based safe zone calculation results.

\section{Discussion}

A method for deriving a multi-dimensional ROM-based safe zone is presented. With this method any arbitrary motion can be considered rather than only pure motions in a single anatomical plane such as in the algorithm presented in (Yoshimine, 2006). Compared to (Widmer \& Zurfluh, 2004), the calculation is simple since no 3D simulation is needed.

The resulting safe zone is multi-dimensional. It does not only specify a suitable cup inclination and anteversion in combination with the stem antetorsion but considers the 3D stem orientation (antetorsion, neutral flexion and adduction) as well as design parameter (head-neck-ratio, neck angle).

The safe zone is highly dependent on the input values for the desired ROM. The specification of a suitable ROM for a specific patient (depending on age, activity level, cultural aspects etc.) is not in the scope of this work but should be investigated in future.

Whereas this work only considered the criterion of prosthesis impingement, further aspects have to be considered for implant design and placement. This includes bony impingement as well as the resulting hip force orientation representing other causes of dislocation (Pedersen, et al., 2005). Moreover, patient-specific musculoskeletal conditions influence the amplitude and orientation of the resulting hip force, edge loading and implant wear and thus should be considered for the definition of an optimal patient-specific implant design and placement. The development of methods for including all relevant criteria for patient-specific safe zone estimation is part of our ongoing work. 


\section{References}

Abdel, M. P. et al., 2016. What safe zone? The vast majority of dislocated THAs are within the Lewinnek safe zone for acetabular component position. Clinical Orthopaedics and Related Research, Volume 474, pp. 386-391.

Biedermann, R. et al., 2005. Reducing the risk of dislocation after total hip arthroplasty THE EFFECT OF ORIENTATION OF THE ACETABULAR COMPONENT. Journal of Bone \& Joint Surgery, British Volume, Volume 87, pp. 762-769.

Lewinnek, G. E. et al., 1978. Dislocations after total hip-replacement arthroplasties.. The Journal of Bone \& Joint Surgery, Volume 60, pp. 217-220.

Nadzadi, M. E. et al., 2003. Kinematics, kinetics, and finite element analysis of commonplace maneuvers at risk for total hip dislocation. Journal of biomechanics, Volume 36, pp. 577-591.

Pedersen, D. R., Callaghan, J. J. \& Brown, T. D., 2005. Activity-dependence of the "safe zone" for impingement versus dislocation avoidance. Medical engineering \& physics, Volume 27, pp. 323328.

Turley, G. A., Ahmed, S. M. Y., Williams, M. A. \& Griffin, D. R., 2011. Establishing a range of motion boundary for total hip arthroplasty. Proceedings of the Institution of Mechanical Engineers, Part H: Journal of Engineering in Medicine, p. 0954411911409306.

Widmer, K.-H. \& Zurfluh, B., 2004. Compliant positioning of total hip components for optimal range of motion. Journal of Orthopaedic Research, Volume 22, pp. 815-821.

$\mathrm{Wu}, \mathrm{G}$. et al., 2002. ISB recommendation on definitions of joint coordinate system of various joints for the reporting of human joint motion - part I: ankle, hip, and spine. Journal of biomechanics, Volume 35, pp. 543-548.

Yoshimine, F., 2006. The safe-zones for combined cup and neck anteversions that fulfill the essential range of motion and their optimum combination in total hip replacements. Journal of biomechanics, Volume 39, pp. 1315-1323.

Yoshimine, F. \& Ginbayashi, K., 2002. A mathematical formula to calculate the theoretical range of motion for total hip replacement. Journal of biomechanics, Volume 35, pp. 989-993. 